\title{
A glycolysis-related gene pairs signature predicts prognosis in patients with hepatocellular carcinoma
}

\author{
Weige Zhou ${ }^{1}$, Shijing Zhang ${ }^{1}$, Zheyou Cai ${ }^{1}$, Fei Gao ${ }^{2}$, Wenhui Deng ${ }^{3}$, Yi Wen ${ }^{4}$, Zhen-wen Qiu ${ }^{4}$, Zheng-kun Hou ${ }^{4}$, \\ Xin-Lin Chen ${ }^{\text {Corresp. } 1}$ \\ ${ }^{1}$ School of Basic Medical Science, Guangzhou University of Chinese Medicine, Guangzhou, China \\ Department of Minimally Invasive \& Interventional Radiology, Sun Yat-sen University Cancer Center, Guangzhou, China \\ 3 The fourth Affiliated Hospital of Guangzhou University of Chinese Medicine, Shenzhen, China \\ 4 The First Affiliated Hospital of Guangzhou University of Chinese Medicine, Guangzhou, China \\ Corresponding Author: Xin-Lin Chen \\ Email address: chenxlsums@126.com
}

Background. Hepatocellular carcinoma (HCC) is one of the most universal malignant liver tumors worldwide. However, there were no systematic studies to establish glycolysis-related gene pairs (GRGPS) signatures for the patients with HCC. Therefore, the study aimed to establish novel GRGPs signatures to better predict the prognosis of HCC.

Methods. Based on the data from Gene Expression Omnibus (GEO), The Cancer Genome Atlas (TCGA) and International Cancer Genome Consortium (ICGC) databases, glycolysis-related mRNAs were used to construct GRGPs. Cox regression was applied to establish a seventeen GRGPs signature in TCGA dataset, which was verified in two validation (European and American, and Asian) datasets.

Results. Seventeen prognostic GRGPs (HMMR_PFKFB1, CHST1_GYS2, MERTK_GYS2, GPC1_GYS2, LDHA_GOT2, IDUA_GNPDA1, IDUA_ME2, IDUA_G6PD, IDUA_GPC1, MPI_GPC1, SDC2_LDHA, PRPS1_PLOD2, GALK1_IER3, MET_PLOD2, GUSB_IGFBP3, IL13RA1_IGFBP3 and CYB5A_IGFBP3) were identified to be significantly progressive factors for the patients with HCC in TCGA dataset, which constituted a GRGPs signature. The patients with HCC were classified into low-risk group and high-risk group based on the GRGPs signature. The GRGPs signature was a significantly independent prognostic indicator for the patients with HCC in TCGA (log-rank $P=2.898 \mathrm{e}-14$ ). Consistent with TCGA dataset, the patients in lowrisk group had a longer OS in two validation datasets (European and American: $P=1.143 e-02$, and Asian: $P=6.342 \mathrm{e}-08$ ). Additionally, the GRGPs signature was also validated as a significantly independent prognostic indicator in two validation datasets.

Conclusion. The seventeen GRGPs and their signature might be molecular biomarkers and therapeutic targets for the patients with HCC. 


\section{A glycolysis-related gene pairs signature predicts prognosis 2 in patients with hepatocellular carcinoma}

3

4 Weige Zhou ${ }^{1}$, Shijing Zhang ${ }^{1}$, Zheyou Cai ${ }^{1}$, Fei Gao ${ }^{2}$, Wenhui Deng ${ }^{3}$, Yi Wen ${ }^{4}$, Zhen-wen Qiu ${ }^{4}$, 5 Zheng-kun $\mathrm{Hou}^{4}$, Xin-lin Chen ${ }^{1}$

1 School of Basic Medical Science, Guangzhou University of Chinese Medicine, Guangzhou, China.

2 Department of Minimally Invasive \& Interventional Radiology, Sun Yat-sen University Cancer Center, Guangzhou, China.

${ }^{3}$ The fourth Affiliated Hospital of Guangzhou University of Chinese Medicine, Shenzhen, China ${ }^{4}$ The First Affiliated Hospital of Guangzhou University of Chinese Medicine, Guangzhou, China

Corresponding Author: Xin-lin Chen

Email address: chenxlsums@126.com (Xin-lin Chen)

\section{ABSTRACT}

Background. Hepatocellular carcinoma (HCC) is one of the most universal malignant liver tumors worldwide. However, there were no systematic studies to establish glycolysis-related gene pairs (GRGPs) signatures for the patients with HCC. Therefore, the study aimed to establish novel GRGPs signatures to better predict the prognosis of HCC.

Methods. Based on the data from Gene Expression Omnibus (GEO), The Cancer Genome Atlas (TCGA) and International Cancer Genome Consortium (ICGC) databases, glycolysis-related mRNAs were used to construct GRGPs. Cox regression was applied to establish a seventeen GRGPs signature in TCGA dataset, which was verified in two validation (European and American, and Asian) datasets.

Results. Seventeen prognostic GRGPs (HMMR_PFKFB1, CHST1_GYS2, MERTK_GYS2, GPC1_GYS2, LDHA_GOT2, IDUA_GNPDA1, IDUA_ME2, IDUA_G6PD, IDUA_GPC1, MPI_GPC1, SDC2_LDHA, PRPS1_PLOD2, GALK1_IER3, MET_PLOD2, GUSB_IGFBP3, IL13RA1_IGFBP3 and CYB5A_IGFBP3) were identified to be significantly progressive factors for the patients with HCC in TCGA dataset, which constituted a GRGPs signature. The patients with HCC were classified into low-risk group and high-risk group based on the GRGPs signature. 
33 The GRGPs signature was a significantly independent prognostic indicator for the patients with 34 HCC in TCGA (log-rank $\mathrm{P}=2.898 \mathrm{e}-14$ ). Consistent with TCGA dataset, the patients in low-risk group had a longer OS in two validation datasets (European and American: $\mathrm{P}=1.143 \mathrm{e}-02$, and Asian: $\mathrm{P}=6.342 \mathrm{e}-08$ ). Additionally, the GRGPs signature was also validated as a significantly independent prognostic indicator in two validation datasets.

Conclusion. The seventeen GRGPs and their signature might be molecular biomarkers and therapeutic targets for the patients with HCC.

Keywords Glycolysis, Hepatocellular carcinoma, GRGPs signature, Prognosis, mRNAs

\section{INTRODUCTION}

Hepatocellular carcinoma (HCC), which is the second most dominant cause of cancer deaths throughout the word, is the most ordinary form of primary carcinoma of the liver (Llovet et al. 2016). It is estimated that approximately 841,000 new cases are expected to occur worldwide and more than 780,000 patients would die of HCC in 2018 (Bray et al. 2018). Kinase and immune checkpoint inhibitors have been shown to be effective options for the treatment of advanced-stage HCC, but they have limited effectiveness (Yang et al. 2019b). Despite the new progress in drug development, the clinical outcomes in patients with advanced HCC remains poor. Based on the Surveillance, Epidemiology, and End Results database, the 5-year survival rate was 30.5\% for patients with local HCC, and less than 5\% for those with distant metastasis (Oweira et al. 2017). Due to the poor outcomes, it is necessary to investigate novel effective markers for the prognosis of HCC.

Recent developments in high-throughput sequencing, technologies and bioinformatics have drastically changed research on genomic in disease, and many marker changes related to prognosis and survival have been revealed through mining databases (Liu et al. 2018). Several biomarkers have been shown to predict the prognosis of the patients with HCC. For example, serum lncRNA urothelial carcinoma-associated 1 (UCA1) was an independent harmful prognostic indicator for HCC (Zheng et al. 2018). Collagen triple helix repeat containing 1 (CTHRC1) may serve as a prognostic biomarker for HCC (Zhou et al. 2019a). Serum acetylcarnitine is a meaningful biomarker reflecting HCC diagnosis and progression (Lu et al. 2016). Notably, a six glycolysisrelated gene signature was found to predict survival in patients with HCC (Jiang et al. 2019). However, in view of the intrinsic biological heterogeneity of tumors and batch effects caused by different sequencing platforms, previous prognostic gene signature had to standardize gene expression profiles, which was very difficult for data processing. Moreover, compared with a single gene marker, multigene prognostic signatures are better alternatives for predicting prognosis 
67 and survival (Chen et al. 2018). Thus, a novel method which omits data standardization and scaling 68 based on the relative ranking of gene expression levels has been used in this study. Many reliable 69 results have been obtained in various studies by using this method (Heinaniemi et al. 2013; Li et al. 2017a; Popovici et al. 2012).

Glycolysis, one of the most ancient metabolic processes, is a low-energy-providing pathway. The metabolic properties of cancer cells differed from those of normal cells (Annibaldi \& Widmann 2010). Cancer cells had rapid metabolic features which increased uptake of glucose and glycolysis (Akram 2013). This allowed cancer cells to preferentially metabolize glucose through aerobic glycolysis, offering them with a progression advantage (Hua et al. 2018). Some studies indicated that aerobic glycolysis phenotype was associated with poor prognosis of HCC (Cui et al. 2018; Guo et al. 2015; Hua et al. 2018; Lin et al. 2018; Xu et al. 2017). However, there were no systematic studies to establish glycolysis-related gene pairs (GRGPs) signatures to predict the survival of patients with HCC. Therefore, it was necessary to establish multigene prognostic signatures for HCC using glycolysis-related genes pairs.

The purpose of this study was to construct GRGPs signatures to predict the prognosis in patients with HCC.

\section{MATERIALS AND METHODS}

\section{Data source}

The expression profiles and clinicopathological data of HCC and normal tissues were obtained from The Cancer Genome Atlas (TCGA, https://portal.gdc.cancer.gov/) and International Cancer Genome Consortium Japan (ICGC, https://dcc.icgc.org/releases/current/Projects/LIRI-JP). The clinical information inclusion criteria were set as follows: (1) patients had completely detailed clinical information; (2) The follow-up time of samples exceeded 30 days. GSE10140, GSE10141, GSE10143, GSE15654, GSE14520, GSE76427, GSE45114 expression profile was derived from the Gene Expression Omnibus (GEO) database, including 1,469 samples. The TCGA was used as training dataset. Other databases were used as validation datasets.

\section{Gene Set Enrichment Analysis}

To explore whether the specific gene sets were significant different between tumor group and normal group, we performed gene sets enrichment analysis (GSEA) (http://www.broadinstitute.org/gsea/index.jsp). The mRNAs expression levels between tumor and non-tumor groups were analyzed to confirm which function could be available for further study. Normalized $P$-value $\leq 0.05$ were considered to be statistically significant.

\section{Construction and evaluation of glycolysis-related gene pairs (GRGPs) signature}


101

102

103

104

105

106

107

108

109

110

111

112

113

114

115

116

117

118

119

120

121

122

123

124

125

126

127

128

129

130

131

132

133

134

135

First of all, the glycolysis-related mRNAs level in the same sample was pairwise compared to generate a score for each GRGP. If the expression level of gene 1 was greater than gene 2, the GRGP score was 1, otherwise it was 0 ( $\mathrm{Li}$ et al. 2017a). GRGPs with high variation in TCGA dataset (median absolute deviation $>0.05$ ) were included in subsequent model construction. The prognostic value of GRGPs was identified by univariate Cox regression. Then, GRGPs with $P \leq$ 0.05 in univariate analysis were incorporated into Lasso regression model in order to establish a GRGPs signature. A risk score was established according to the following formula: risk score $=$ $\sum_{i}$ Coefficient $\left(\mathrm{GRGP}_{i}\right) * \operatorname{Score}\left(\mathrm{GRGP}_{i}\right)$. To classify patients into low-risk group and high-risk group, the optimal cut off of the GRGPs signature was defined using time-dependent receiver operating characteristic (ROC) curve analysis at 1 year in the TCGA dataset for overall survival (OS). The OS between two groups was compared utilizing Kaplan-Meier and Log-rank test. Risk score and other clinicopathological characteristics were included in the model so as to confirm whether risk score was an independent factor to predict the progress of the patients. Further, the clinical value of the GRGPs signature was verified by comparing the risk scores of patients with different ages, gender, grade and stage.

The OS of the patients with $\mathrm{HCC}$ at 1 year, 3 years and 5 years were predicted using a nomogram. Index of concordance (C-index) and Calibration curves were applied to explore the accuracy of the model established from TCGA dataset.

\section{Validation of the GRGPs signature}

Seven GEO databases (GSE10140, GSE10141, GSE10143, GSE15654, GSE14520, GSE76427, and GSE45114) and ICGC database were enrolled for subsequent verification. According to human race, all the databases were divided into European and American dataset and Asian dataset. European and American dataset (765 samples) included GSE10140, GSE10141, GSE10143, and GSE15654 database; Asian dataset (947 samples) included GSE14520, GSE76427, GSE45114 and ICGC database. Due to the lack of detailed clinical information (such as age, gender, stage) in European and American dataset, thus it was only used for survival analysis. Asian dataset was used for subsequent survival analysis and independent prognostic analysis. The GRGPs signature was calculated, and then Kaplan-Meier, Log-rank test and Cox regression were used to verify whether the GRGPs signature was significantly associated with OS. The ROC curve, C-index and Calibration curves were constructed to examine whether the model established by TCGA dataset could effectively predict survival in patients with HCC.

\section{Statistical analysis}

Cox regression was utilized to evaluate the associations between the expression levels of the glycolytic-related mRNAs and OS. Moreover, univariate and multivariate Cox regression were applied to determine the prognostic values of the GRGPs signature and various clinicopathological 
136

137

138

139

140

141

142

143

144

145

146

147

148

149

150

151

152

153

154

155

156

157

158

159

160

161

162

163

164

165

166

167

168

169

170

characteristics. The prediction accuracy of the risk score for 1-year, 3-years and 5-years survival was evaluated using ROC curve analysis. Statistical tests were two-sided, and $P$ values below 0.05 were considered to be statistically significant.

\section{RESULTS}

\section{Preliminary selection of genes using GSEA}

According to inclusion criteria, 349 patients with HCC were obtained. Expression signatures of marker gene sets were obtained by condensing multiple gene sets from the Molecular Signature Database (MSigDB). Each expression signature involved 50 specific gene sets that stand for clearly defined biological statuses and processes (Zhang et al. 2019a). GSEA was applied to investigate whether the identified gene sets revealed significant differences between the tumor and normal groups. Forty-four gene sets were upregulated in $\mathrm{HCC}$, and 20 gene sets were highly enriched, with normalized $P<0.05$ among the 50 gene sets (Fig. 1, Table S1). As can be seen in the Table S1, the biological process of glycolysis $(P<0.05)$ contained 199 genes, which was the second largest in size.

\section{Identification of prognostic GRGPs}

Based on 199 GRGs, 19,701 GRGPs were established. After removing GRGPs with comparatively low variation (MAD>0.05), 1,102 GRGPs were reserved and selected as initial candidate GRGPs. Cox regression was utilized to select prognostic GRGPs. According to the results of univariate Cox, 35 GRGPs had prognostic values for the patients with HCC $(P<0.05$, Table. S2). Subsequently, lasso regression model revealed that 17 GRGPs as prognostic factors (Table 1, Figs. 2A-2B). As shown in Table 1, five GRGPs (HMMR_PFKFB1, CHST1_GYS2, MERTK_GYS2, GPC1_GYS2 and LDHA_GOT2) were found to be harmful prognostic factors and twelve GRGPs (IDUA_GNPDA1, IDUA_ME2, IDUA_G6PD, IDUA_GPC1, MPI_GPC1, SDC2_LDHA, PRPS1_PLOD2, GALK1_IER3, MET_PLOD2, GUSB_IGFBP3, IL13RA1_IGFBP3 and CYB5A_IGFBP3) were verified to be beneficial prognostic factors.

\section{Construction of GRGPs signature}

These seventeen GRGPs were utilized to establish a GRGPs signature. Risk score of GRGPs signature for each patient was calculated utilizing the following formula (formula 1): risk score = $(-0.30794 *$ IDUA_GNPDA1 $)-(0.15299 *$ IDUA_ME2 $)-(0.16389 *$ IDUA_G6PD $)-(0.35599$ * IDUA_GPC1 $)+(0.04846 *$ HMMR_PFKFB1 $)-(0.35632 *$ MPI_GPC1 $)-(0.29752 *$ SDC2_LDHA $)-(0.09077 *$ PRPS1_PLOD2 $)-(0.06137 *$ GALK1_IER3 $)+(0.02511 *$ CHST1_GYS2 $)-(0.26287 *$ MET_PLOD2 $)-(0.00305 *$ GUSB_IGFBP3 $)+(0.34302 *$ MERTK_GYS2 $)+(0.20608 *$ GPC1_GYS2 $)-(0.31484 *$ IL13RA1_IGFBP3 $)+(0.17629 *$ LDHA_GOT2 $)-(0.10962 *$ CYB5A_IGFBP3). The cutoff point of risk score was set at -0.698 
171

172

173

174

175

176

177

178

179

180

181

182

183

184

185

186

187

188

189

190

191

192

193

194

195

196

197

198

199

200

201

202

203

204

205

utilizing ROC curve analysis, which classified the patients into high-risk group or low-risk group (Figs. 2C). Risk score was significantly associated with OS of the patients with HCC, in which OS in low-risk group was longer than that in high-risk group $(P=2.898 \mathrm{e}-14$, Fig. $3 \mathrm{~A})$. The survival time of patients with HCC decreased with risk score increasing (Fig. 4).

\section{Association between risk score and clinicopathologic factors}

Risk score increased with age, stage, and survival status, demonstrating that the GRGPs signature might be relevant to the progression of HCC. Risk score of patients with advanced-stage and advanced-age were significantly higher than those with early-stage and early-age (Figs. S1A-1B). Risk scores of dead patients were higher than those of living patients (Fig. S1C). The heat map illustrated that the high expression of these seventeen GRGPs were significantly related to female, lower survival status of patients, higher stage and higher grade (Fig. S1D).

\section{The GRGPs signature was an independent prognostic factor}

In TCGA dataset, univariate Cox regression revealed that GRGPs signature was associated with OS and its HR was 3.508 (95\% CI: 2.608-4.720, $P<0.001$, Table S3, Fig. 5A). After controlling clinical features such as gender, age, tumor stage and grade, GRGPs signature remained to be an independent prognostic indicator $(\mathrm{HR}=3.204,95 \% \mathrm{CI}=2.293-4.476, P<0.001$, Table 2, Fig. 5B). GRGPs signature and TNM stage were independent prognostic factors based on the TCGA. Thus, these factors were included in nomogram. GRGPs signature was the largest contributor to 1-year, 3-year and 5-year OS (Figs. 6A). The C-index of the nomogram was 0.764 (95\% CI: 0.715 0.813). The areas under the ROC curve (AUC) corresponding to the survival at 1 year, 3 years and 5 years were $0.803,0.777$ and 0.774 , respectively $(P<0.05)$. The $\mathrm{C}$-index, ROC curve and Calibration explained that the GRGPs signature had better accuracy (Figs. 6B-6D).

\section{Validation of the GRGPs signature}

In validation datasets, the risk score of GRGPs signature was calculated according to formula 1. The risk score was also significantly correlated with OS of patients with HCC (European and American dataset: $P=1.143 \mathrm{e}-02$, Fig. 3B; Asian dataset: $P=6.342 \mathrm{e}-08$, Fig. $3 \mathrm{C}$ ). Univariate independent prognostic analysis indicated that GRGPs signature were independent prognostic factors in Asian dataset (HR of risk score $=2.661,95 \%$ CI: 1.862-3.803, $P<0.001$, Table S4, Fig. 5C). After controlling stage and gender, GRGPs signature remained an independent prognostic indicator in multivariate analysis $(\mathrm{HR}=2.567,95 \% \mathrm{CI}=1.714-3.844, P<0.001$, Table S5, Fig. 5D). According to results of independent prognostic analysis, GRGPs signature, stage and gender were included in nomogram based on Asian dataset. GRGPs signature and age were the largest contributor to 1-year, 3-year and 5-year OS in Asian dataset nomogram (Figs. 6E). The C-index of nomogram based on Asian dataset was 0.705 (95\% CI: 0.660-0.750). The areas under the ROC curve at 1 year, 3 years and 5 years in Asian dataset were 0.694, 0.664, 0.536, respectively (Fig. 
206

207

208

209

210

211

212

213

214

215

216

217

218

219

220

221

222

223

224

225

226

227

228

229

230

231

232

233

234

235

236

237

238

239

240

$6 \mathrm{H})$. The three-year and five-year calibration curves also proved that the GRGPs signature had great accuracy and robustness (Figs. 6F-6G).

\section{DISCUSSION}

$\mathrm{HCC}$ is one of the most universal malignant liver tumors worldwide. Long-term prognosis for HCC remains mighty poor, with metastasis being the major cause of mortality (Uchino et al. 2011). Most tumor cells support synthetic growth and evade apoptosis through aerobic glycolysis. (Iansante et al. 2015; Warburg 1956). Glycolytic transcriptional factors and glycolysis-related proteins in cancers are significantly correlated with poor prognosis, indicating that glycolytic status may be potentially valuable prognostic biomarkers for various cancers (Yu et al. 2019). Thus, it was valuable and urgent to establish a GRGPs signature in term of predicting the prognosis of patients with $\mathrm{HCC}$.

In consideration of the intrinsic biological heterogeneity of tumors and batch effects caused by different sequencing platforms, previous gene signature needed to standardize or scale gene expression profiles, which resulted in the inability to process large amounts of data quickly. The construction method of prognostic model using gene pairs in this study can overcome these shortcomings greatly, which has been shown to have high stability and accuracy in some studies (Heinaniemi et al. 2013; Li et al. 2017a; Popovici et al. 2012).

In this study, a GRGPs signature was constructed to predict the prognosis of patients with HCC, whose accuracy was better than gene signature previously constructed (Jiang et al. 2019). The HCC patients from TCGA dataset could be classified into low-risk group and high-risk group using the optimal cutoff point determined by ROC curve $(P=2.898 \mathrm{e}-14)$. The high-risk patients with HCC had shorter OS than the low-risk patients. Consistent with TCGA dataset, The OS in low-risk patients was longer than that in high-risk patients in two validation datasets. The AUC in TCGA and validation datasets were integrally greater than 0.6 which revealed that the GRGPs signature had certain accuracy in predicting survival. Both univariate and multivariate analysis indicated that the GRGPs signature could be set as an independent prognostic factor to predict the prognosis of patients with HCC in TCGA dataset and validation dataset. Furthermore, nomograms were established based on the GRGPs signature and other clinical characteristics, which might serve as potential predictive tools for patients with HCC.

Seventeen GRGPs (HMMR_PFKFB1, CHST1_GYS2, MERTK_GYS2, GPC1_GYS2, LDHA_GOT2, IDUA_GNPDA1, IDUA_ME2, IDUA_G6PD, IDUA_GPC1, MPI_GPC1, SDC2_LDHA, PRPS1_PLOD2, GALK1_IER3, MET_PLOD2, GUSB_IGFBP3, IL13RA1_IGFBP3 and CYB5A_IGFBP3) were associated to OS of patients with HCC, which might be molecular markers of prognosis and potential therapeutic targets. 
241

242

243

244

245

246

247

248

249

250

251

252

253

254

255

256

257

258

259

260

261

262

263

264

265

266

267

268

269

270

271

272

273

274

275

276

The prognostic signature consists of 17 GRGPs including 23 unique GRGs. Most of the GRGs involved in this signature are metabolism and tumor related genes that play an important role in patient prognosis and tumor metabolism. GYS is the rate-limiting enzyme for glycogen synthesis, which consists of two isoforms: GYS1 and GYS2 (Roach et al. 2012; Szymanska et al. 2015). Previous studies revealed that loss of GYS2 leaded to glycogen storage disease type 0 (GSD-0) with the symptom of weakened glucose tolerance (Orho et al. 1998; Szymanska et al. 2015). A recent study indicated that decreased expression levels of GYS2 reduced glycogen and significantly correlated with metastasis and poor prognosis of the patients with HCC, GYS2 restricted HBV-Related HCC growth through negative feedback loop with p53 (Chen et al. 2019). Savci-Heijink et al. demonstrated that the expression level of IDUA was down-regulated in patients with breast cancer and IDUA could be used as one of the potential targets for distinguishing whether breast cancer patients will undergo visceral metastasis (Savci-Heijink et al. 2019). A common growth factor co-receptor, Glypican-1 (GPC1), is abnormally rich in pancreatic cancer and that GPC1 deficiency inhibits tumor growth, angiogenesis and metastasis (Aikawa et al. 2008). Enhanced expression level of GPC1 is associated with BMP and activin receptors in pancreatic cancer, and the low expression of GPC1 could suppress pancreatic cancer cell growth (Kayed et al. 2006). It was found that increased expression level of GPC1 was significantly relevant with poor prognosis of the patients with pancreatic ductal adenocarcinoma (Lu et al. 2017; Zhou et al. 2018). The overexpression of GPC1 was correlated with poor prognosis of the patients with oesophageal squamous cell carcinoma, and GPC1 is a key molecule that alters the threshold of chemoresistance to chemo-sensitivity against cis-Diammineplatinum (II) dichloride (CDDP) (Hara et al. 2016). However, there were no studies to report GPC1 prognostic role in HCC. Based on the results of this study, it was revealed to be potential molecular biomarkers and therapeutic targets for the HCC patients. Thus, more researches were necessary to figure out how GPC1 affects the prognosis of HCC exactly. In this study, HMMR was considered as potential molecular target for the treatment of HCC. HMMR has not been directly reported as therapeutic target for HCC. The Y-linked proto-oncogene could promote the expression of HMMR, which was correlated with poor prognosis in the patients with HCC(Kido \& Lau 2019). Over-expression of HMMR was verified as indicators of poor prognosis and metastasis in lung cancer (Liu et al. 2019; Zhang et al. 2019a). HMMR was confirmed to be a potential independent indicator of predicting survival in patients with papillary muscle-invasive bladder cancer (Wang et al. 2019c; Yang et al. 2019a).

Several limitations exist in the current study. First, the study was a retrospective study, although we tried to incorporate as many datasets as possible to verify this signature more rigorously. More prospective studies was demanded to prove the prognostic functions of glycolysis-related signals. Second, Gene expression signatures are susceptible to sampling deviation caused by intratumor heterogeneity. Although we removed low variation GRGPs so as to reduce cross-study batch 
277 effects, some may still reserve genetic heterogeneity. Third, the functional experiments should be 278 conducted to further indicate the potential molecular mechanisms for predicting effects of GRGPs.

279 In conclusion, our study systematically demonstrated the expression of glycolysis-related 280 mRNAs in HCC, verifying the prognostic value of these mRNAs. The GRGPs signature could 281 predict survival in patients with HCC. Therefore, the seventeen GRGPs and their signature may 282 be molecular biomarkers and therapeutic targets for the patients with HCC, which conduces to 283 explore new treatments for HCC.

\section{ADDITIONAL INFORMATION AND DECLARATIONS}

288

\section{Funding}

This study was funded by the National Natural Science Foundation of China (81774451), the Natural Science Foundation of Guangdong Province (2017A030313827), and Science Program for Overseas Scholar (Xinhuo plan) of Guangzhou University of Chinese Medicine (XH20190102).

\section{Competing Interests}

The authors declare there are no competing interests.

\section{Author Contributions}

-Weige Zhou conceived and designed the experiments, performed the experiments, analyzed and interpreted the data, contributed reagents/materials/analysis tools, composed or reviewed drafts of the paper, read and approved the final draft.

-Shijing Zhang conducted experiments, analyzed and interpreted the data, wrote or reviewed drafts of the dissertation, read and approved the final draft.

-Zheyou Cai conducted experiments, analyzed and interpreted the data contribute dreagents/materials/analysis tools, read and approved the final draft.

-Fei Gao conducted experiments, helped to modify the draft, read and approved the final draft.

-Wenhui Deng performed the experiments, wrote drafts of the dissertation, read and approved the final draft.

-Yi Wen performed the experiments, read and approved the final draft.

-Zhen-wen Qiu performed the experiments, read and approved the final draft.

-Zheng-kun Hou performed the experiments, read and approved the final draft.

-Xin-lin Chen conceived and designed the experiments, modify the draft, read and approved the final draft. 
Data Availability

The following information was provided according to data availability:

Data is available at NCBI GEO under accession number GSE10140, GSE10141, GSE10143, GSE15654, GSE14520, GSE76427, and GSE45114.All analyzed or generated data were incorporated in this published paper and its supplementary information file. 
351

352

353

354

355

356

357

358

359

360

361

362

363

364

365

366

367

368

369

370

371

372

373

374

375

376

377

378

379

380

381

382

383

384

385

386

387

388

389

390

391

Zhao Y, and He X. 2015. MiR-199a-5p is negatively associated with malignancies and regulates glycolysis and lactate production by targeting hexokinase 2 in liver cancer. Hepatology 62:1132-1144. 10.1002/hep.27929

Hara H, Takahashi T, Serada S, Fujimoto M, Ohkawara T, Nakatsuka R, Harada E, Nishigaki T, Takahashi Y, Nojima S, Miyazaki Y, Makino T, Kurokawa Y, Yamasaki M, Miyata H, Nakajima K, Takiguchi S, Morii E, Mori M, Doki Y, and Naka T. 2016. Overexpression of glypican-1 implicates poor prognosis and their chemoresistance in oesophageal squamous cell carcinoma. Br J Cancer 115:66-75. 10.1038/bjc.2016.183

Heinaniemi M, Nykter M, Kramer R, Wienecke-Baldacchino A, Sinkkonen L, Zhou JX, Kreisberg R, Kauffman SA, Huang S, and Shmulevich I. 2013. Gene-pair expression signatures reveal lineage control. Nat Methods 10:577-583. 10.1038/nmeth.2445

Hua S, Lei L, Deng L, Weng X, Liu C, Qi X, Wang S, Zhang D, Zou X, Cao C, Liu L, and Wu D. 2018. miR-139-5p inhibits aerobic glycolysis, cell proliferation, migration, and invasion in hepatocellular carcinoma via a reciprocal regulatory interaction with ETS1. Oncogene 37:1624-1636. 10.1038/s41388-017-0057-3

Iansante V, Choy PM, Fung SW, Liu Y, Chai JG, Dyson J, Del Rio A, D'Santos C, Williams R, Chokshi S, Anders RA, Bubici C, and Papa S. 2015. PARP14 promotes the Warburg effect in hepatocellular carcinoma by inhibiting JNK1-dependent PKM2 phosphorylation and activation. Nat Commun 6:7882. 10.1038/ncomms8882

Jiang L, Zhao L, Bi J, Guan Q, Qi A, Wei Q, He M, Wei M, and Zhao L. 2019. Glycolysis gene expression profilings screen for prognostic risk signature of hepatocellular carcinoma. Aging (Albany NY) 11:10861-10882. 10.18632/aging.102489

Kayed H, Kleeff J, Keleg S, Jiang X, Penzel R, Giese T, Zentgraf H, Buchler MW, Korc M, and Friess H. 2006. Correlation of glypican-1 expression with TGF-beta, BMP, and activin receptors in pancreatic ductal adenocarcinoma. Int J Oncol 29:1139-1148.

Kido T, and Lau YC. 2019. The Y-linked proto-oncogene TSPY contributes to poor prognosis of the male hepatocellular carcinoma patients by promoting the pro-oncogenic and suppressing the anti-oncogenic gene expression. Cell Biosci 9:22. 10.1186/s13578-0190287-X

Li B, Cui Y, Diehn M, and Li R. 2017a. Development and Validation of an Individualized Immune Prognostic Signature in Early-Stage Nonsquamous Non-Small Cell Lung Cancer. JAMA Oncol 3:1529-1537. 10.1001/jamaoncol.2017.1609

Li L, Guo L, Wang Q, Liu X, Zeng Y, Wen Q, Zhang S, Kwok HF, Lin Y, and Liu J. 2017b. DAPK1 as an independent prognostic marker in liver cancer. PeerJ 5:e3568. $10.7717 /$ peerj. 3568

Li X, Xu W, Kang W, Wong SH, Wang M, Zhou Y, Fang X, Zhang X, Yang H, Wong CH, To KF, Chan SL, Chan MTV, Sung JJY, Wu WKK, and Yu J. 2018. Genomic analysis of liver cancer unveils novel driver genes and distinct prognostic features. Theranostics 8:1740-1751. 10.7150/thno.22010

Li Y, Zhu Z, Zhang S, Yu D, Yu H, Liu L, Cao X, Wang L, Gao H, and Zhu M. 2011. ShRNAtargeted centromere protein A inhibits hepatocellular carcinoma growth. PLoS One 
6:e17794. 10.1371/journal.pone.0017794

Lin YH, Wu MH, Huang YH, Yeh CT, Cheng ML, Chi HC, Tsai CY, Chung IH, Chen CY, and Lin KH. 2018. Taurine up-regulated gene 1 functions as a master regulator to coordinate glycolysis and metastasis in hepatocellular carcinoma. Hepatology 67:188-203. 10.1002/hep.29462

Liu C, Li Y, Wei M, Zhao L, Yu Y, and Li G. 2019. Identification of a novel glycolysis-related gene signature that can predict the survival of patients with lung adenocarcinoma. Cell Cycle 18:568-579. 10.1080/15384101.2019.1578146

Liu S, Miao C, Liu J, Wang CC, and Lu XJ. 2018. Four differentially methylated gene pairs to predict the prognosis for early stage hepatocellular carcinoma patients. J Cell Physiol 233:6583-6590. 10.1002/jcp.26256

Llovet JM, Zucman-Rossi J, Pikarsky E, Sangro B, Schwartz M, Sherman M, and Gores G. 2016. Hepatocellular carcinoma. Nat Rev Dis Primers 2:16018. 10.1038/nrdp.2016.18

Lu H, Niu F, Liu F, Gao J, Sun Y, and Zhao X. 2017. Elevated glypican-1 expression is associated with an unfavorable prognosis in pancreatic ductal adenocarcinoma. Cancer Med 6:1181-1191. 10.1002/cam4.1064

Lu Y, Li N, Gao L, Xu YJ, Huang C, Yu K, Ling Q, Cheng Q, Chen S, Zhu M, Fang J, Chen $\mathrm{M}$, and Ong CN. 2016. Acetylcarnitine Is a Candidate Diagnostic and Prognostic Biomarker of Hepatocellular Carcinoma. Cancer Res 76:2912-2920. 10.1158/00085472.CAN-15-3199

Ma X, Zhou L, and Zheng S. 2020. Transcriptome analysis revealed key prognostic genes and microRNAs in hepatocellular carcinoma. PeerJ 8:e8930. 10.7717/peerj.8930

Metodieva SN, Nikolova DN, Cherneva RV, Dimova, II, Petrov DB, and Toncheva DI. 2011. Expression analysis of angiogenesis-related genes in Bulgarian patients with early-stage non-small cell lung cancer. Tumori 97:86-94.

Orho M, Bosshard NU, Buist NR, Gitzelmann R, Aynsley-Green A, Blumel P, Gannon MC, Nuttall FQ, and Groop LC. 1998. Mutations in the liver glycogen synthase gene in children with hypoglycemia due to glycogen storage disease type 0. J Clin Invest 102:507515. 10.1172/JCI2890

Oweira H, Petrausch U, Helbling D, Schmidt J, Mehrabi A, Schob O, Giryes A, and AbdelRahman O. 2017. Prognostic value of site-specific extra-hepatic disease in hepatocellular carcinoma: a SEER database analysis. Expert Rev Gastroenterol Hepatol 11:695-701. 10.1080/17474124.2017.1294485

Philips KB, Kurtoglu M, Leung HJ, Liu H, Gao N, Lehrman MA, Murray TG, and Lampidis TJ. 2014. Increased sensitivity to glucose starvation correlates with downregulation of glycogen phosphorylase isoform PYGB in tumor cell lines resistant to 2-deoxy-D-glucose. Cancer Chemother Pharmacol 73:349-361. 10.1007/s00280-013-2358-8

Popovici V, Budinska E, Tejpar S, Weinrich S, Estrella H, Hodgson G, Van Cutsem E, Xie T, Bosman FT, Roth AD, and Delorenzi M. 2012. Identification of a poor-prognosis BRAF-mutant-like population of patients with colon cancer. J Clin Oncol 30:1288-1295. 10.1200/JCO.2011.39.5814 
Roach PJ, Depaoli-Roach AA, Hurley TD, and Tagliabracci VS. 2012. Glycogen and its metabolism: some new developments and old themes. Biochemical Journal 441:763-787. 10.1042/Bj20111416

Savci-Heijink CD, Halfwerk H, Koster J, Horlings HM, and van de Vijver MJ. 2019. A specific gene expression signature for visceral organ metastasis in breast cancer. $B M C$ Cancer 19:333. 10.1186/s12885-019-5554-z

Song X, Du R, Gui H, Zhou M, Zhong W, Mao C, and Ma J. 2020. Identification of potential hub genes related to the progression and prognosis of hepatocellular carcinoma through integrated bioinformatics analysis. Oncol Rep 43:133-146. 10.3892/or.2019.7400

Szymanska E, Rokicki D, Watrobinska U, Ciara E, Halat P, Ploski R, and Tylki-Szymanka A. 2015. Pediatric patient with hyperketotic hypoglycemia diagnosed with glycogen synthase deficiency due to the novel homozygous mutation in GYS2. Molecular Genetics and Metabolism Reports 4:83-86. 10.1016/j.ymgmr.2015.07.003

Terashima M, Fujita Y, Togashi Y, Sakai K, De Velasco MA, Tomida S, and Nishio K. 2014. KIAA1199 interacts with glycogen phosphorylase kinase beta-subunit (PHKB) to promote glycogen breakdown and cancer cell survival. Oncotarget 5:7040-7050. 10.18632/oncotarget.2220

Uchino K, Tateishi R, Shiina S, Kanda M, Masuzaki R, Kondo Y, Goto T, Omata M, Yoshida H, and Koike K. 2011. Hepatocellular carcinoma with extrahepatic metastasis: clinical features and prognostic factors. Cancer 117:4475-4483. 10.1002/cncr.25960

Wang L, Huang J, Jiang M, and Lin H. 2012. Tissue-specific transplantation antigen P35B (TSTA3) immune response-mediated metabolism coupling cell cycle to postreplication repair network in no-tumor hepatitis/cirrhotic tissues (HBV or HCV infection) by biocomputation. Immunol Res 52:258-268. 10.1007/s12026-012-8337-z

Wang M, Wang L, Wu S, Zhou D, and Wang X. 2019a. Identification of Key Genes and Prognostic Value Analysis in Hepatocellular Carcinoma by Integrated Bioinformatics Analysis. Int J Genomics 2019:3518378. 10.1155/2019/3518378

Wang T, Fu X, Jin T, Zhang L, Liu B, Wu Y, Xu F, Wang X, Ye K, Zhang W, and Ye L. 2019b. Aspirin targets P4HA2 through inhibiting NF-kappaB and LMCD1-AS1/let-7g to inhibit tumour growth and collagen deposition in hepatocellular carcinoma. EBioMedicine 45:168-180. 10.1016/j.ebiom.2019.06.048

Wang Y, Chen L, Ju L, Qian K, Liu X, Wang X, and Xiao Y. 2019c. Novel Biomarkers Associated With Progression and Prognosis of Bladder Cancer Identified by Co-expression Analysis. Front Oncol 9:1030. 10.3389/fonc.2019.01030

Wang Y, Wu J, Xu J, and Lin S. 2019d. Clinical significance of high expression of stanniocalcin2 in hepatocellular carcinoma. Biosci Rep 39. 10.1042/BSR20182057

Wang Z, Yin B, Wang B, Ma Z, Liu W, and Lv G. 2013. MicroRNA-210 promotes proliferation and invasion of peripheral nerve sheath tumor cells targeting EFNA3. Oncol Res 21:145154. 10.3727/096504013X13841340689573

Warburg O. 1956. On the origin of cancer cells. Science 123:309-314. 10.1126/science.123.3191.309 
Wu Y, Yao N, Feng Y, Tian Z, Yang Y, and Zhao Y. 2019. Identification and characterization of sexual dimorphismlinked gene expression profile in hepatocellular carcinoma. Oncol Rep 42:937-952. 10.3892/or.2019.7217

Xu Q, Tu J, Dou C, Zhang J, Yang L, Liu X, Lei K, Liu Z, Wang Y, Li L, Bao H, Wang J, and Tu K. 2017. HSP90 promotes cell glycolysis, proliferation and inhibits apoptosis by regulating PKM2 abundance via Thr-328 phosphorylation in hepatocellular carcinoma. Mol Cancer 16:178. 10.1186/s12943-017-0748-y

Yang D, Ma Y, Zhao P, Ma J, and He C. 2019a. Systematic screening of protein-coding gene expression identified HMMR as a potential independent indicator of unfavorable survival in patients with papillary muscle-invasive bladder cancer. Biomed Pharmacother 120:109433. 10.1016/j.biopha.2019.109433

Yang JD, Hainaut P, Gores GJ, Amadou A, Plymoth A, and Roberts LR. 2019b. A global view of hepatocellular carcinoma: trends, risk, prevention and management. Nat Rev Gastroenterol Hepatol 16:589-604. 10.1038/s41575-019-0186-y

Yu M, Chen S, Hong W, Gu Y, Huang B, Lin Y, Zhou Y, Jin H, Deng Y, Tu L, Hou B, and Jian Z. 2019. Prognostic role of glycolysis for cancer outcome: evidence from 86 studies. J Cancer Res Clin Oncol 145:967-999. 10.1007/s00432-019-02847-w

Yu Z, Zhou X, Wang W, Deng W, Fang J, Hu H, Wang Z, Li S, Cui L, Shen J, Zhai L, Peng S, Wong J, Dong S, Yuan Z, Ou G, Zhang X, Xu P, Lou J, Yang N, Chen P, Xu RM, and Li G. 2015. Dynamic phosphorylation of CENP-A at Ser68 orchestrates its cell-cycledependent deposition at centromeres. Dev Cell 32:68-81. 10.1016/j.devcel.2014.11.030

Zhang L, Zhang Z, and Yu Z. 2019a. Identification of a novel glycolysis-related gene signature for predicting metastasis and survival in patients with lung adenocarcinoma. J Transl Med 17:423. 10.1186/s12967-019-02173-2

Zhang X, Zhuang R, Ye Q, Zhuo J, Chen K, Lu D, Wei X, Xie H, Xu X, and Zheng S. 2019b. High Expression of Human AugminComplex Submit 3 Indicates Poor Prognosis and Associates with Tumor Progression in Hepatocellular Carcinoma. J Cancer 10:1434-1443. $10.7150 /$ jca. 28317

Zhang ZH, Wu YG, Qin CK, Rong ZH, Su ZX, and Xian GZ. 2014. Stanniocalcin 2 expression predicts poor prognosis of hepatocellular carcinoma. Oncol Lett 8:2160-2164. 10.3892/ol.2014.2520

Zheng ZK, Pang C, Yang Y, Duan Q, Zhang J, and Liu WC. 2018. Serum long noncoding RNA urothelial carcinoma-associated 1: A novel biomarker for diagnosis and prognosis of hepatocellular carcinoma. $J$ Int Med Res 46:348-356. 10.1177/0300060517726441

Zhou CY, Dong YP, Sun X, Sui X, Zhu H, Zhao YQ, Zhang YY, Mason C, Zhu Q, and Han SX. 2018. High levels of serum glypican-1 indicate poor prognosis in pancreatic ductal adenocarcinoma. Cancer Med 7:5525-5533. 10.1002/cam4.1833

Zhou H, Su L, Liu C, Li B, Li H, Xie Y, and Sun D. 2019a. CTHRC1 May Serve As A Prognostic Biomarker For Hepatocellular Carcinoma. Onco Targets Ther 12:7823-7831. 10.2147/OTT.S219429

Zhou Y, Jin Z, and Wang C. 2019b. Glycogen phosphorylase B promotes ovarian cancer 
515

516

517

518

519

520

521

522

523

524

525

526

527

528

529

530

531

532

533

534

535

536

537

538

539

540

541

542

543

544

545

546

547

548

549

550

progression via Wnt/beta-catenin signaling and is regulated by miR-133a-3p. Biomed Pharmacother 120:109449. 10.1016/j.biopha.2019.109449

\section{Figure and Table legend}

Figure. 1 Enrichment graph of five gene sets with significant differences between tumor and non-tumor tissues. The high expression of these genes was principally enriched in biological processes such as glycolysis, DNA repair, metabolism and protein synthesis secretion.

Figure. 2 GRGPs selection utilizing lasso model based on TCGA dataset. (A) Elastic net regularization course with partial likelihood deviance plot. The vertical dashed line with minimum partial likelihood deviance value is at the optimal logarithmic (Lambda) value. Lambda is the parameter which controls the regulation degree of lasso regression complexity. The ordinate is the value of the coefficient, the lower abscissa is log (lambda), and the upper abscissa is the number of non-zero coefficients in the model. (B) Lasso coefficient values of 17 prognosis GRGPs. Each colored line represents the change track of each independent variable coefficient. (C) Timedependent ROC curve for GRGPs at 1 year. GRGPs score of -0.698 was utilized as cutoff point for GRGPs.

Figure. 3 The Kaplan-Meier (KM) survival curves of the GRGPs signature for patients with HCC based on TCGA and two validation datasets. (A) The KM survival curves of TCGA dataset demonstrated that high-risk group had shorter OS period contrasted with low-risk group $(P$ $<0.001$ ). (B-C) Consistent with TCGA dataset, the OS of patients in high-risk group was shorter than that in low-risk group in two validation datasets $(P<0.05)$.

Figure. 4 The GRGPs signature analysis of patients with HCC in TCGA dataset. (A) The lowrisk group and high-risk group for the GRGPs signature in patients with HCC. (B) The survival status and time of patients with HCC. (C) Visualized heat map of the seventeen vital prognosis GRGPs expression in patients with HCC. The color from green to red reveals a rising tendency from low to high levels.

Figure. 5 Prognostic indicators based on GRGPs signature revealed great clinical values in TCGA dataset and Asian dataset. Univariate (A) and multivariate (B) Cox regression of the relevancy between clinical features (containing the risk score) and OS of patients with HCC in 
551 TCGA dataset. Univariate (C) and multivariate (D) Cox regression of the relevancy between

552 clinical indicators (including the risk score) and OS of patients with HCC in Asian dataset.

553

554

555

556

557

558

559

560

561

562

563

564

565

566

567

568

569

570

571

572

573

574

575

576

577

578

579

580

581

582

583

584

585

586

Figure. 6 The evaluation of prognostic GRGPs signature in TCGA dataset and Asian dataset. (A and E) The nomogram figure about 1-year, 3-year or 5-year OS in HCC based on TCGA dataset (A) or Asian dataset (E). The point in the nomogram represented the individual score of each variable under different values. Total points represented the sum of the individual scores corresponding to all variables. For a single variable, we could get the corresponding point by drawing a vertical line upward, which must be perpendicular to the point line. For example, if someone's risk score is - 1, the corresponding point of risk score in nomogram based on TCGA dataset was about 42.5 by drawing a vertical line upward. Similarly, the corresponding point of the third stage was about 16.5. Then, add the points of all variables to get the total points of the patient (59). Based on the total point, the corresponding 1-year survival rate of the patient was about 0.86. (B, C, F and G) Calibration plots of 3-year (B) and 5-year (C) based on TCGA dataset. Calibration plots of 3-year (F) and 5-year (G) based on Asian dataset. Calibration plots for evaluating the agreement between the predicted and the actual OS for the model established by GRGPs. The $45^{\circ}$ reference line indicates perfect calibration, where the predicted probabilities are consistent with the actual probabilities. (D and $\mathrm{H}$ ) The areas under the ROC curve corresponding to 1 year, 3 years and 5 years of survival in TCGA (D) or Asian datasets (H). The higher area under the ROC curve meant greater model accuracy.

Figure. S1 Clinicopathological significance of the prognostic factors of HCC in TCGA and Asian datasets. Statistical $P$ values were significant at (A) age, (B) survival status, (C) TNM stage, (D) The heat map revealed the distribution of clinicopathological features and seventeen GRGPs expression contrasted between the low-risk and high-risk groups in TCGA dataset.

Table 1 Lasso regression coefficients and molecular function information of seventeen GRGPs based on TCGA-HCC data.

Table 2 Clinical characteristics and risk score of HCC utilizing multivariate cox regression in TCGA dataset.

Table S1 The results of Gene set enrichment analysis was based on the difference in gene expression between tumor and normal groups (Top 20 p-value).

Table S2 Results of Univariate Cox about GRGPs based on TCGA-HCC data. 
587

588 Table S3 Clinical features and risk score of $\mathrm{HCC}$ utilizing univariate cox regression in TCGA 589 dataset.

590

591 Table S4 Clinical features and risk score of HCC using univariate cox regression in Asian 592 dataset.

593 Table S5 Clinical characteristics and risk score of HCC using multivariate cox regression in 594 Asian dataset. 
Figure 1

Enrichment graph of five gene sets with significant differences between tumor and nontumor tissues.

The high expression of these genes was principally enriched in biological processes such as glycolysis, DNA repair, metabolism and protein synthesis secretion.

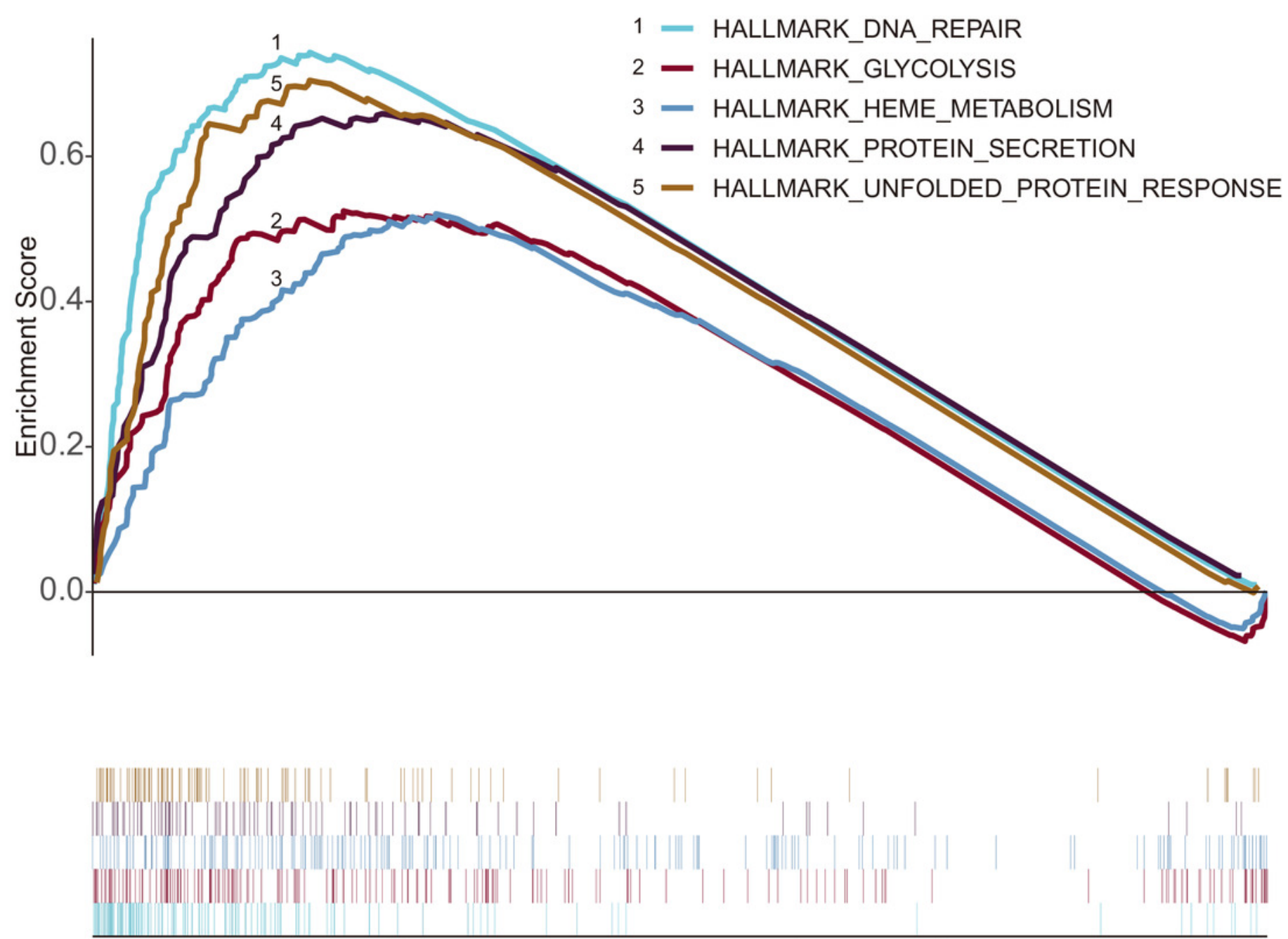

high expression<------------>low expression 


\section{Figure 2}

GRGPs selection utilizing lasso model based on TCGA dataset.

(A) Elastic net regularization course with partial likelihood deviance plot. The vertical dashed line with minimum partial likelihood deviance value is at the optimal logarithmic (Lambda) value. Lambda is the parameter which controls the regulation degree of lasso regression complexity. The ordinate is the value of the coefficient, the lower abscissa is log (lambda), and the upper abscissa is the number of non-zero coefficients in the model. (B) Lasso coefficient values of 17 prognosis GRGPs. Each colored line represents the change track of each independent variable coefficient. (C) Time-dependent ROC curve for GRGPs at 1 year. GRGPs score of -0.698 was utilized as cutoff point for GRGPs. 


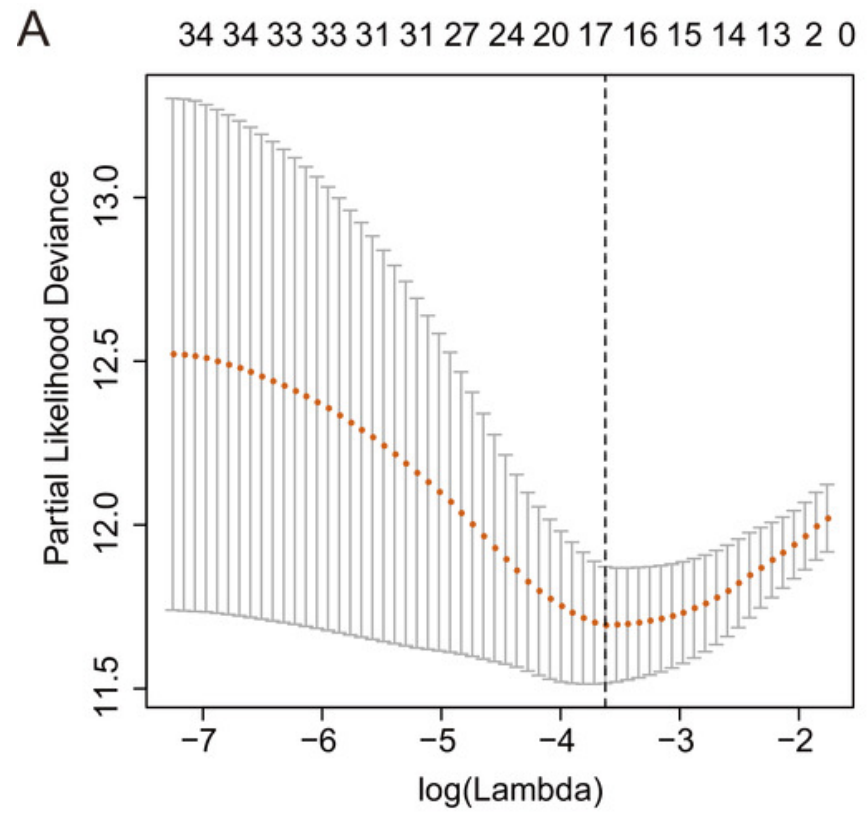

B

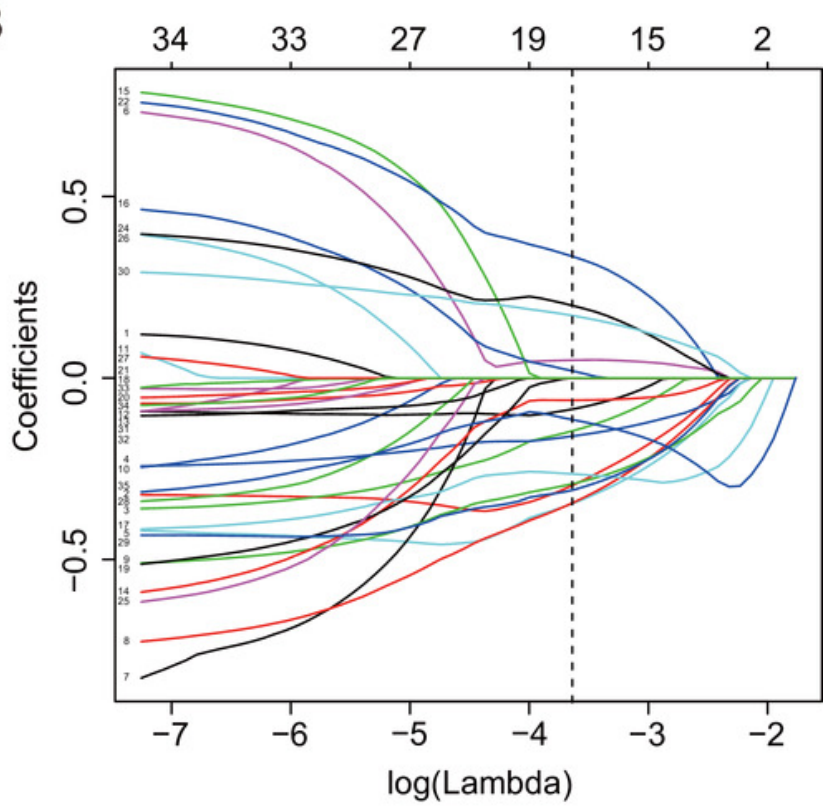

C

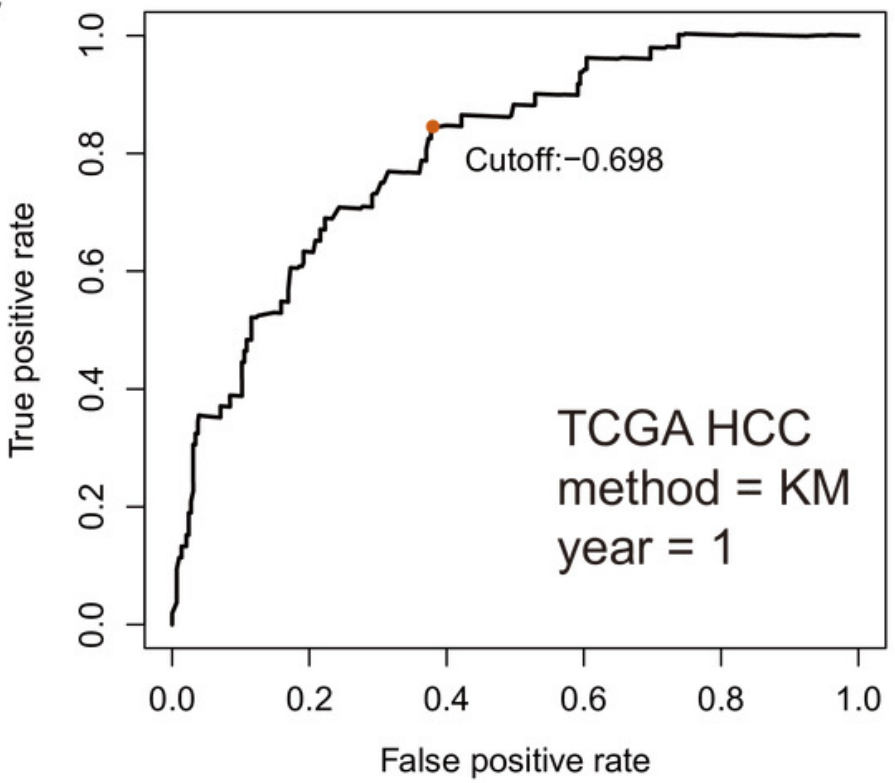


Figure 3

The Kaplan-Meier (KM) survival curves of the GRGPs signature for patients with HCC based on TCGA and two validation datasets.

(A) The KM survival curves of TCGA dataset demonstrated that high-risk group had shorter OS period contrasted with low-risk group $(P<0.001)$. (B-C) Consistent with TCGA dataset, the OS of patients in high-risk group was shorter than that in low-risk group in two validation datasets $(P<0.05)$.

A

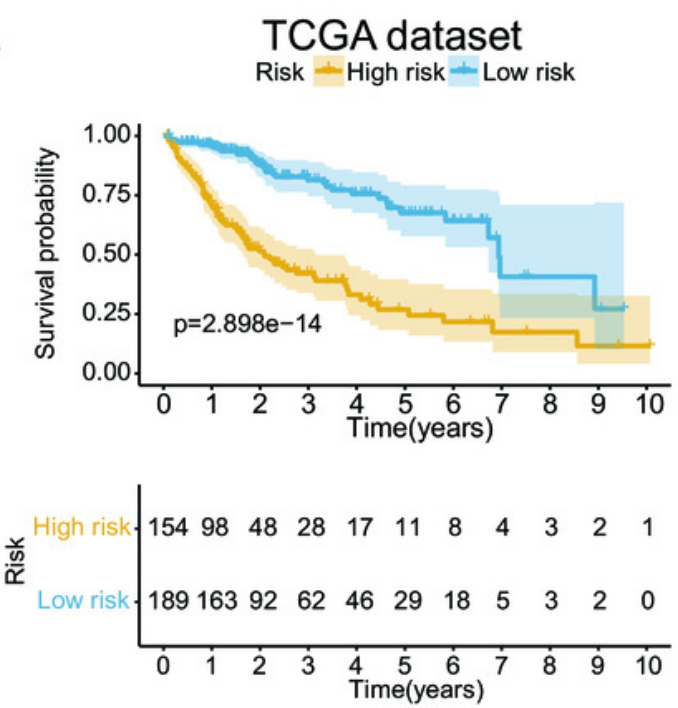

C
B
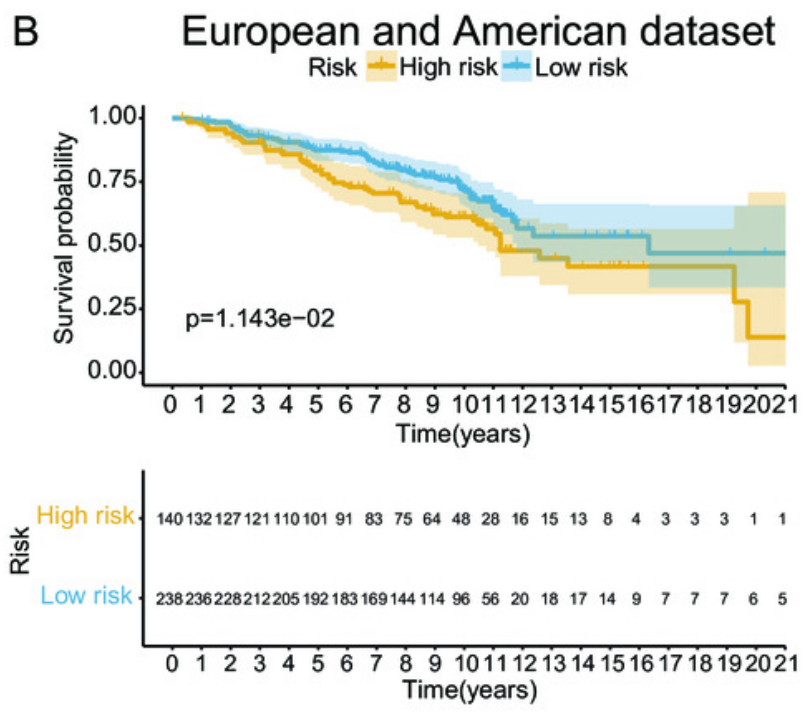

Asian dataset

Risk High risk Low risk
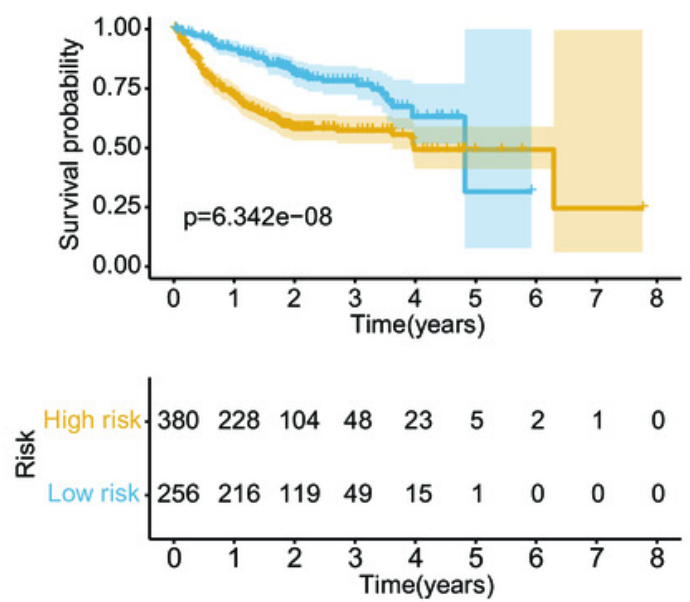


\section{Figure 4}

The GRGPs signature analysis of patients with HCC in TCGA dataset.

(A) The low-risk group and high-risk group for the GRGPs signature in patients with HCC. (B) The survival status and time of patients with HCC. (C) Visualized heat map of the seventeen vital prognosis GRGPs expression in patients with HCC. The color from green to red reveals a rising tendency from low to high levels. 


\section{TCGA dataset}

A

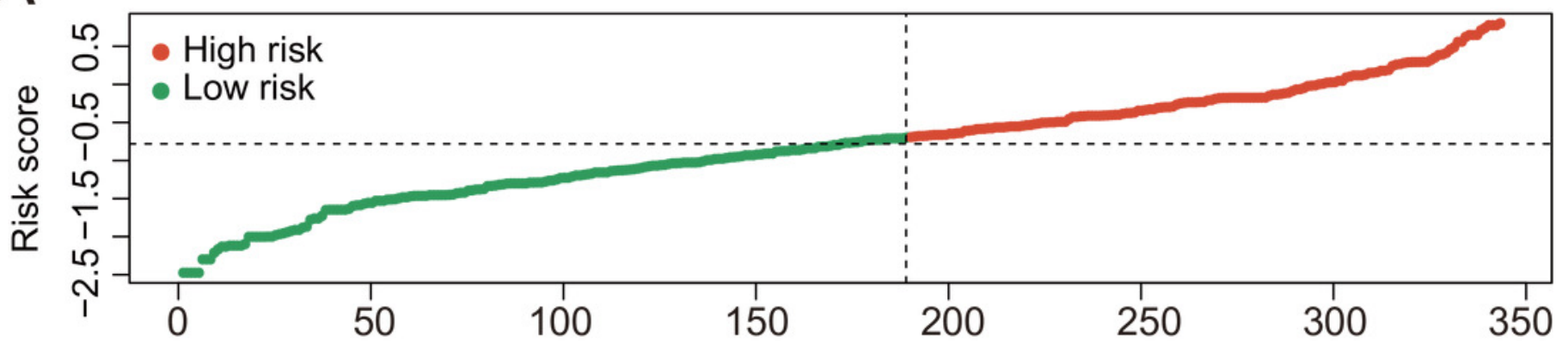

Patients (increasing risk socre)

B

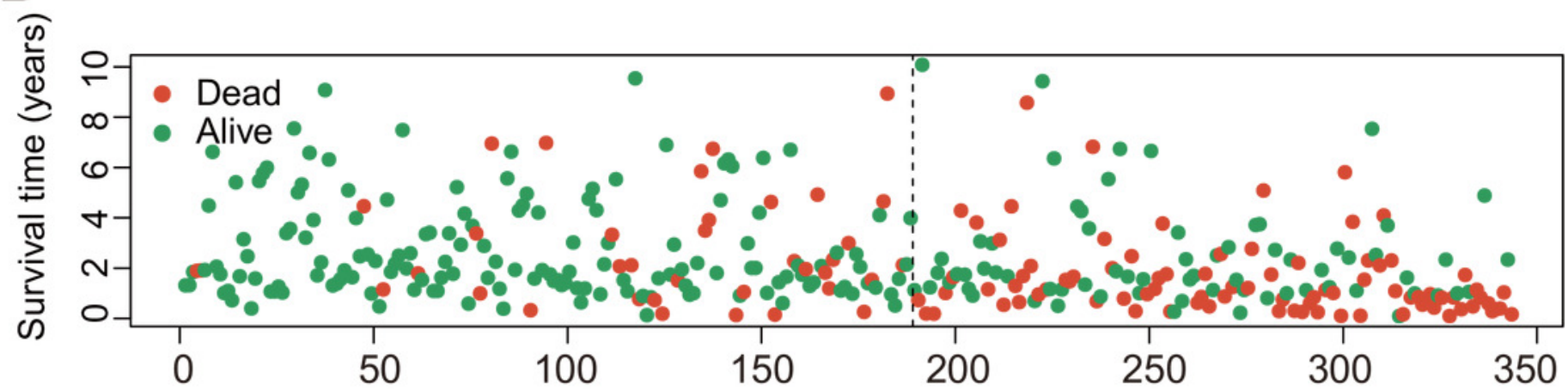

Patients (increasing risk socre)

C

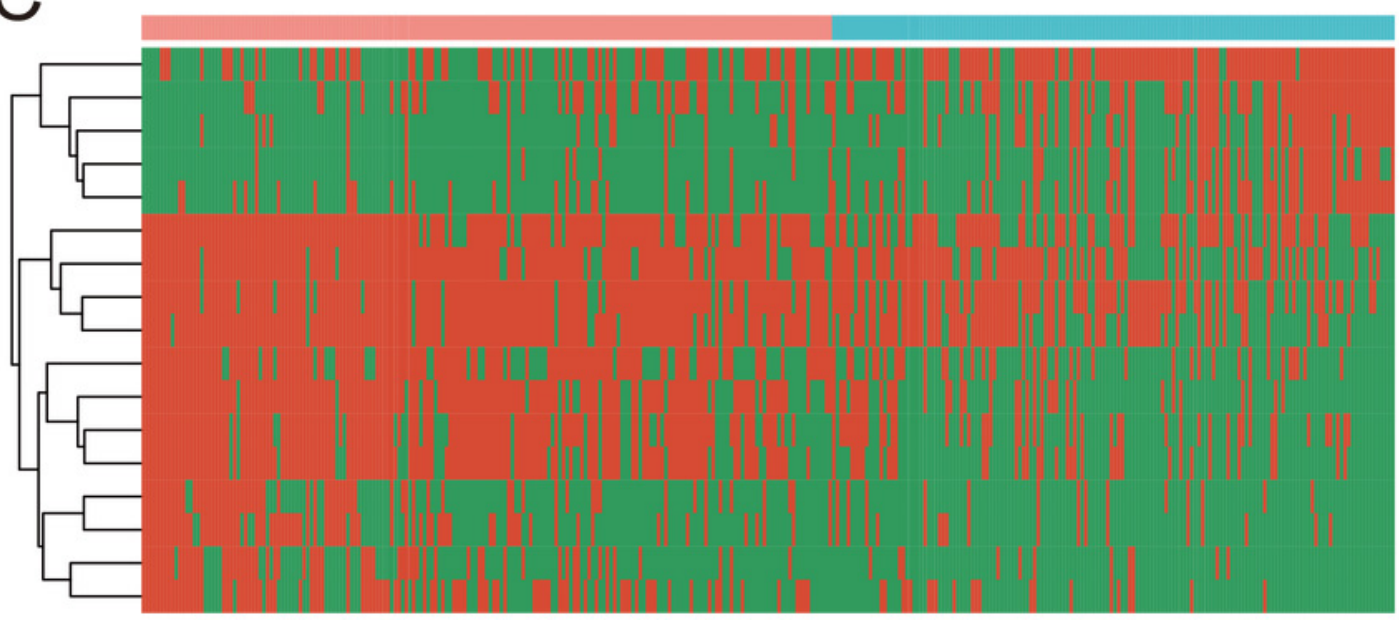

LDHA|GOT2

GPC1|GYS2

HMMR|PFKFB1

CHST1|GYS2

MERTK|GYS2

IDUA|ME2

GALK1|IER3

PRPS1|PLOD2

MET|PLOD2

SDC2|LDHA

CYB5A|IGFBP3 0

GUSB|IGFBP3

IL13RA1|IGFBP3

IDUA|GPC1

MPI|GPC1

IDUA|GNPDA1

IDUA|G6PD 


\section{Figure 5}

Prognostic indicators based on GRGPs signature revealed great clinical values in TCGA dataset and Asian dataset.

Univariate (A) and multivariate (B) Cox regression of the relevancy between clinical features (containing the risk score) and OS of patients with HCC in TCGA dataset. Univariate (C) and multivariate (D) Cox regression of the relevancy between clinical indicators (including the risk score) and OS of patients with HCC in Asian dataset.

A

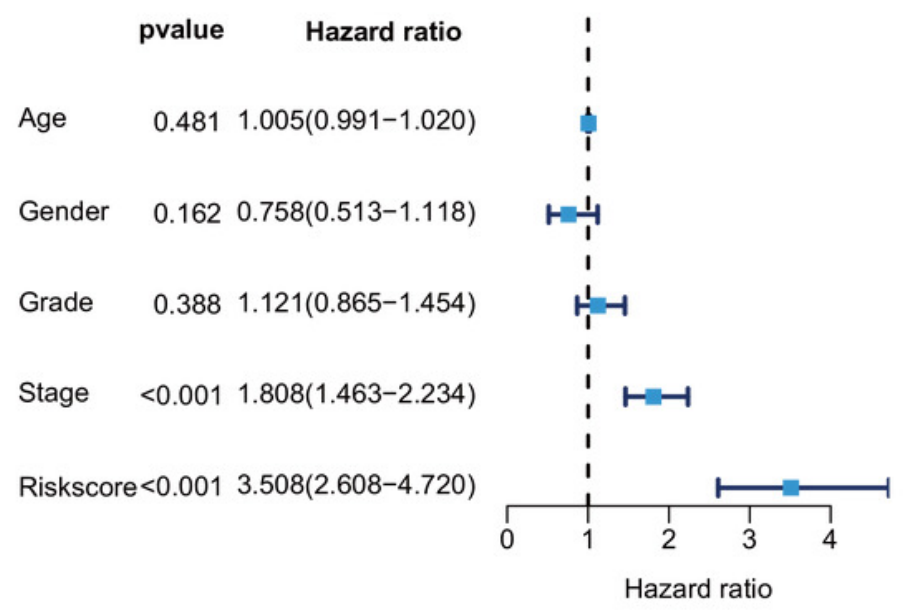

B

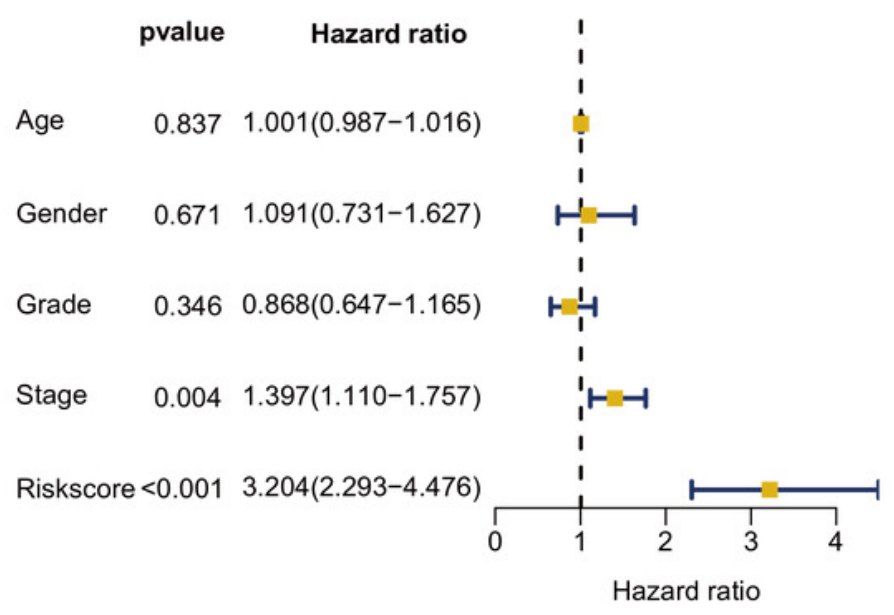

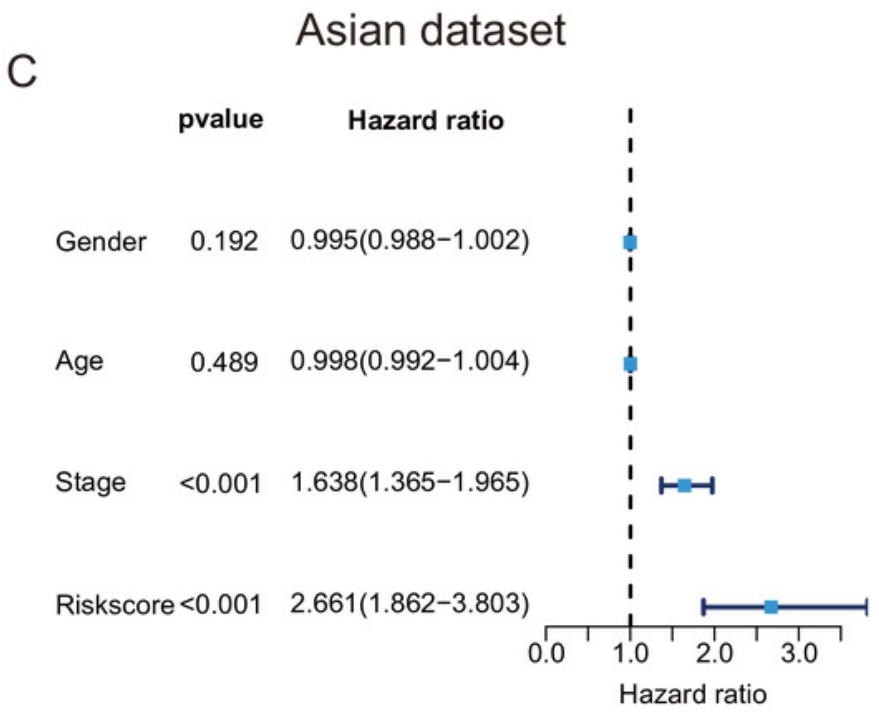

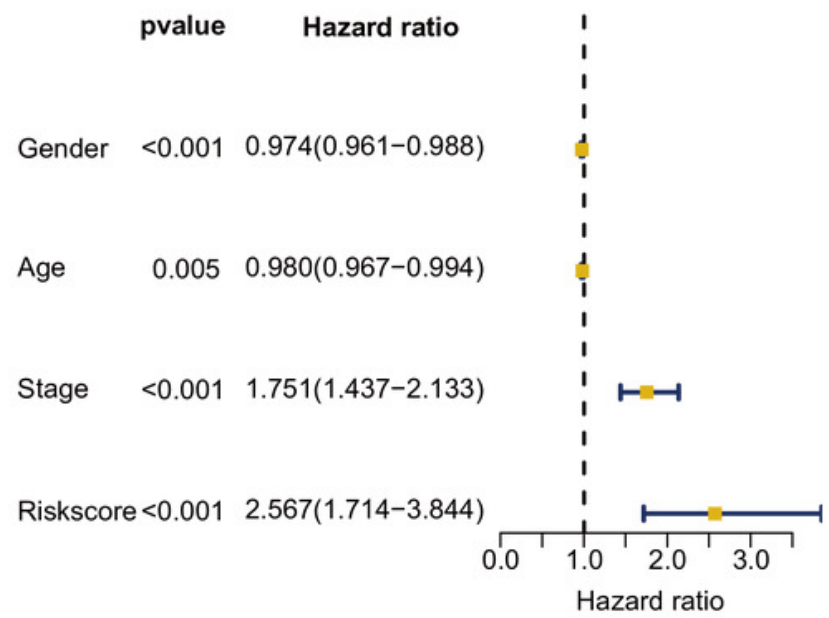




\section{Figure 6}

The evaluation of prognostic GRGPs signature in TCGA dataset and Asian dataset.

( $A$ and $E$ ) The nomogram figure about 1-year, 3-year or 5-year OS in HCC based on TCGA dataset (A) or Asian dataset (E). The point in the nomogram represented the individual score of each variable under different values. Total points represented the sum of the individual scores corresponding to all variables. For a single variable, we could get the corresponding point by drawing a vertical line upward, which must be perpendicular to the point line. For example, if someone's risk score is - 1, the corresponding point of risk score in nomogram based on TCGA dataset was about 42.5 by drawing a vertical line upward. Similarly, the corresponding point of the third stage was about 16.5. Then, add the points of all variables to get the total points of the patient (59). Based on the total point, the corresponding 1-year survival rate of the patient was about 0.86. (B, C, F and G) Calibration plots of 3-year (B) and 5-year (C) based on TCGA dataset. Calibration plots of 3-year (F) and 5-year (G) based on Asian dataset. Calibration plots for evaluating the agreement between the predicted and the actual OS for the model established by GRGPs. The $45^{\circ}$ reference line indicates perfect calibration, where the predicted probabilities are consistent with the actual probabilities. (D and $\mathrm{H}$ ) The areas under the ROC curve corresponding to 1 year, 3 years and 5 years of survival in TCGA (D) or Asian datasets (H). The higher area under the ROC curve meant greater model accuracy. 
A

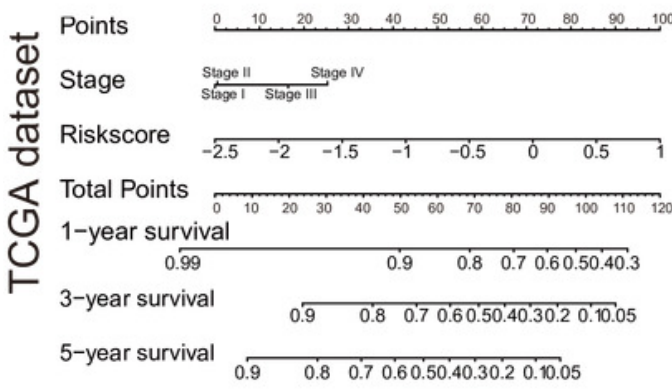

E

Points

¿. Gender

๘ Age

范 Stage

Riskscore

(大) Total Points $-1 . \overline{6-1.4-1.2-1}-0.8-0.6-0.4-0.2 \quad 0 \quad 0.20 .4 \quad 0.60 .8$

ज) 1-year survival

$\varangle$ 3-year survival

5-year survival
B

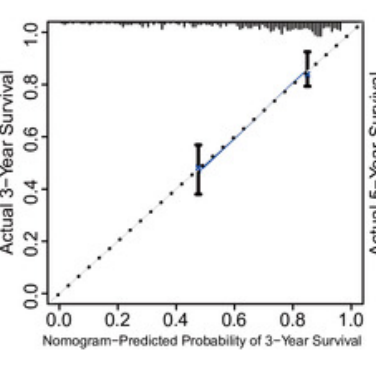

$\mathrm{F}$

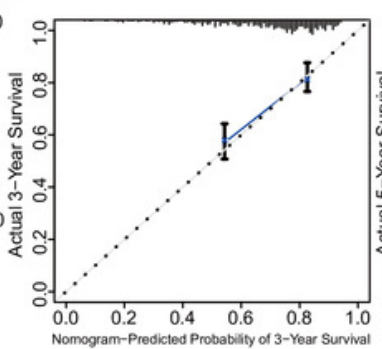

C

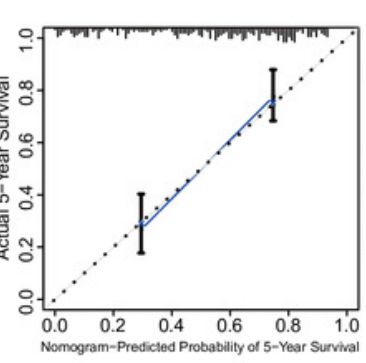

G

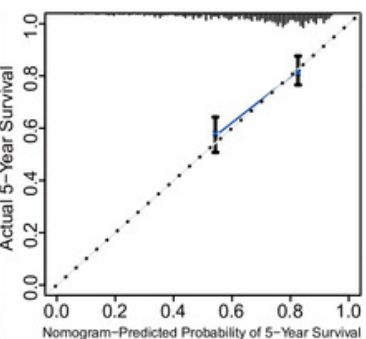

D

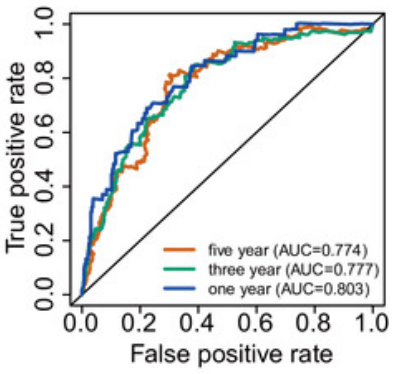

$\mathrm{H}$

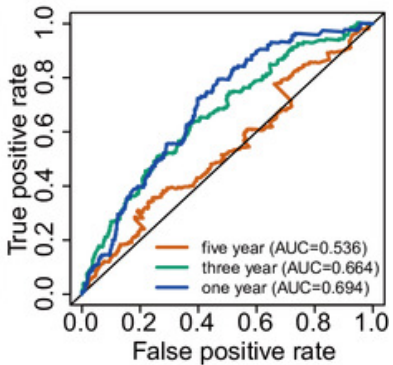




\section{Table $\mathbf{1}$ (on next page)}

Lasso regression coefficients and molecular function information of seventeen GRGPs based on TCGA-HCC data. 


\begin{tabular}{|c|c|c|c|c|c|c|}
\hline Gene1 & Encoding protein & Function & Gene2 & Encoding protein & Function & Coefficient \\
\hline IDUA & $\begin{array}{l}\text { hydrolyzes the terminal alpha-L- } \\
\text { iduronic acid residues of two } \\
\text { glycosaminoglycans, dermatan } \\
\text { sulfate and heparan sulfate. }\end{array}$ & $\begin{array}{l}\text { Chondroitin sulfate/dermatan } \\
\text { sulfate metabolism and } \\
\text { Glycosaminoglycan } \\
\text { metabolism. }\end{array}$ & GNPDA1 & An allosteric enzyme & $\begin{array}{l}\text { The reversible conversion of } \\
\text { D-glucosamine-6-phosphate } \\
\text { into D-fructose-6-phosphate } \\
\text { and ammonium }\end{array}$ & -0.294 \\
\hline IDUA & $\begin{array}{l}\text { hydrolyzes the terminal alpha-L- } \\
\text { iduronic acid residues of two } \\
\text { glycosaminoglycans, dermatan } \\
\text { sulfate and heparan sulfate. }\end{array}$ & $\begin{array}{c}\text { Chondroitin sulfate/dermatan } \\
\text { sulfate metabolism and } \\
\text { Glycosaminoglycan } \\
\text { metabolism. }\end{array}$ & ME2 & $\begin{array}{l}\text { A mitochondrial NAD- } \\
\text { dependent malic enzyme }\end{array}$ & $\begin{array}{c}\text { Catalyzes the oxidative } \\
\text { decarboxylation of malate to } \\
\text { pyruvate }\end{array}$ & -0.145 \\
\hline IDUA & $\begin{array}{l}\text { hydrolyzes the terminal alpha-L- } \\
\text { iduronic acid residues of two } \\
\text { glycosaminoglycans, dermatan } \\
\text { sulfate and heparan sulfate. }\end{array}$ & $\begin{array}{l}\text { Chondroitin sulfate/dermatan } \\
\text { sulfate metabolism and } \\
\text { Glycosaminoglycan } \\
\text { metabolism. }\end{array}$ & G6PD & $\begin{array}{c}\text { A cytosolic enzyme } \\
\text { encoded by a } \\
\text { housekeeping X-linked } \\
\text { gene }\end{array}$ & Produce NADPH & -0.160 \\
\hline IDUA & $\begin{array}{l}\text { hydrolyzes the terminal alpha-L- } \\
\text { iduronic acid residues of two } \\
\text { glycosaminoglycans, dermatan } \\
\text { sulfate and heparan sulfate. }\end{array}$ & $\begin{array}{l}\text { Chondroitin sulfate/dermatan } \\
\text { sulfate metabolism and } \\
\text { Glycosaminoglycan } \\
\text { metabolism. }\end{array}$ & GPC1 & $\begin{array}{l}\text { Disease related genes } \\
\text { belongs to the glypican } \\
\text { family }\end{array}$ & $\begin{array}{l}\text { Play a role in the control of } \\
\text { cell division and growth } \\
\text { regulation. }\end{array}$ & -0.345 \\
\hline HMMR & Involved in cell motility. & $\begin{array}{c}\text { Regulation of PLK1 Activity } \\
\text { at G2/M Transition and } \\
\text { Metabolism. }\end{array}$ & PFKFB1 & $\begin{array}{l}\text { A member of the family } \\
\text { of bifunctional 6- } \\
\text { phosphofructo-2-kinase }\end{array}$ & $\begin{array}{l}\text { An activator of the } \\
\text { glycolysis pathway and an } \\
\text { inhibitor of the } \\
\text { gluconeogenesis pathway/ } \\
\text { participate in hepatocellular } \\
\text { carcinoma tumorigenesis. }\end{array}$ & 0.049 \\
\hline MPI & $\begin{array}{l}\text { Phosphomannose isomerase } \\
\text { catalyzes the interconversion of } \\
\text { fructose-6-phosphate and } \\
\text { mannose-6-phosphate }\end{array}$ & $\begin{array}{l}\text { Metabolism of proteins and } \\
\text { Amino sugar and nucleotide } \\
\text { sugar metabolism. }\end{array}$ & GPC1 & $\begin{array}{l}\text { Disease related genes } \\
\text { belongs to the glypican } \\
\text { family }\end{array}$ & $\begin{array}{c}\text { Play a role in the control of } \\
\text { cell division and growth } \\
\text { regulation. }\end{array}$ & -0.343 \\
\hline $\mathrm{SDC} 2$ & $\begin{array}{c}\text { A transmembrane (type I) } \\
\text { heparan sulfate proteoglycan and }\end{array}$ & $\begin{array}{l}\text { Microglia Activation During } \\
\text { Neuroinflammation: Overview }\end{array}$ & LDHA & $\begin{array}{l}\text { Cancer-related protein } \\
\text { belongs to the }\end{array}$ & $\begin{array}{l}\text { Catalyzes the conversion of } \\
\text { L-lactate and NAD to }\end{array}$ & -0.291 \\
\hline
\end{tabular}




\begin{tabular}{|c|c|c|c|c|c|c|}
\hline & $\begin{array}{l}\text { is a member of the syndecan } \\
\text { proteoglycan family. }\end{array}$ & $\begin{array}{l}\text { and Cell surface interactions at } \\
\text { the vascular wall. }\end{array}$ & & LDH/MDH superfamily & $\begin{array}{l}\text { pyruvate and NADH in the } \\
\text { final step of anaerobic } \\
\text { glycolysis. }\end{array}$ & \\
\hline PRPS1 & $\begin{array}{l}\text { Catalyzes the } \\
\text { phosphoribosylation of ribose 5- } \\
\text { phosphate to 5-phosphoribosyl- } \\
\text { 1-pyrophosphate }\end{array}$ & $\begin{array}{c}\text { Thiopurine Pathway, } \\
\text { Pharmacokinetics/Pharmacody } \\
\text { namics and Carbon } \\
\text { metabolism. }\end{array}$ & PLOD2 & $\begin{array}{l}\text { A membrane-bound } \\
\text { homodimeric enzyme }\end{array}$ & $\begin{array}{c}\text { Participate in collagen chain } \\
\text { trimerization and } \\
\text { degradation of the } \\
\text { extracellular matrix }\end{array}$ & -0.085 \\
\hline GALK1 & $\begin{array}{l}\text { Galactokinase is a major enzyme } \\
\text { for the metabolism of galactose. }\end{array}$ & $\begin{array}{l}\text { Galactokinase is a major } \\
\text { enzyme for the metabolism of } \\
\text { galactose. }\end{array}$ & IER3 & $\begin{array}{l}\text { A predicted intracellular } \\
\text { protein belongs to the } \\
\text { IER3 family }\end{array}$ & $\begin{array}{l}\text { Protect cells from Fas- or } \\
\text { tumor necrosis factor type } \\
\text { alpha-induced apoptosis }\end{array}$ & -0.061 \\
\hline CHST1 & $\begin{array}{l}\text { A member of the keratin } \\
\text { sulfotransferase family of } \\
\text { proteins. The encoded enzyme } \\
\text { catalyzes the sulfation of the } \\
\text { proteoglycan keratin. }\end{array}$ & $\begin{array}{l}\text { Among its related pathways } \\
\text { are Keratan sulfate/keratin } \\
\text { metabolism and Metabolism. }\end{array}$ & GYS2 & Liver glycogen synthase & $\begin{array}{l}\text { Participate in galactose } \\
\text { metabolism and glycogen } \\
\text { metabolism. }\end{array}$ & 0.019 \\
\hline MET & $\begin{array}{l}\text { A member of the receptor } \\
\text { tyrosine kinase family of } \\
\text { proteins and the product of the } \\
\text { proto-oncogene MET. }\end{array}$ & $\begin{array}{l}\text { Hepatocyte growth factor, } \\
\text { induces dimerization and } \\
\text { activation of the receptor. }\end{array}$ & PLOD2 & $\begin{array}{l}\text { A membrane-bound } \\
\text { homodimeric enzyme }\end{array}$ & $\begin{array}{c}\text { Participate in collagen chain } \\
\text { trimerization and } \\
\text { degradation of the } \\
\text { extracellular matrix }\end{array}$ & -0.265 \\
\hline MERTK & $\begin{array}{c}\text { A member of the } \\
\text { MER/AXL/TYRO3 receptor } \\
\text { kinase family. }\end{array}$ & $\begin{array}{l}\text { Regulate cell survival, } \\
\text { migration, differentiation, and } \\
\text { phagocytosis of apoptotic } \\
\text { cells. }\end{array}$ & GYS2 & Liver glycogen synthase & $\begin{array}{l}\text { Participate in galactose } \\
\text { metabolism and glycogen } \\
\text { metabolism }\end{array}$ & 0.334 \\
\hline GPC1 & $\begin{array}{l}\text { Disease related genes belongs to } \\
\text { the glypican family. }\end{array}$ & $\begin{array}{l}\text { Play a role in the control of } \\
\text { cell division and growth } \\
\text { regulation. }\end{array}$ & GYS2 & Liver glycogen synthase & $\begin{array}{l}\text { Participate in galactose } \\
\text { metabolism and glycogen } \\
\text { metabolism }\end{array}$ & 0.198 \\
\hline IL13RA1 & $\begin{array}{l}\text { A subunit of the interleukin } 13 \\
\text { receptor }\end{array}$ & $\begin{array}{c}\text { Bind tyrosine kinase TYK2 } \\
\text { and mediate the signaling } \\
\text { processes }\end{array}$ & IGFBP3 & $\begin{array}{l}\text { Encodes a protein with an } \\
\text { IGFBP domain and a } \\
\text { thyroglobulin type-I }\end{array}$ & $\begin{array}{l}\text { Prolonging the half-life of } \\
\text { insulin-like growth factor } \\
\text { (IGF) and altering their }\end{array}$ & -0.308 \\
\hline
\end{tabular}




\begin{tabular}{|c|c|c|c|c|c|c|}
\hline & & & & domain. & $\begin{array}{l}\text { interaction with cell surface } \\
\text { receptors }\end{array}$ & \\
\hline LDHA & $\begin{array}{l}\text { Cancer-related protein belongs to } \\
\text { the LDH/MDH superfamily }\end{array}$ & $\begin{array}{l}\text { Catalyzes the conversion of L- } \\
\text { lactate and NAD to pyruvate } \\
\text { and NADH in the final step of } \\
\text { anaerobic glycolysis }\end{array}$ & GOT2 & $\begin{array}{l}\text { A pyridoxal phosphate- } \\
\text { dependent enzyme }\end{array}$ & $\begin{array}{l}\text { Play a role in amino acid } \\
\text { metabolism and the urea and } \\
\text { tricarboxylic acid cycles. }\end{array}$ & 0.171 \\
\hline CYB5A & A membrane-bound cytochrome & $\begin{array}{c}\text { Reduces ferric hemoglobin } \\
\text { (methemoglobin) to ferrous } \\
\text { hemoglobin }\end{array}$ & IGFBP3 & $\begin{array}{l}\text { Encodes a protein with an } \\
\text { IGFBP domain and a } \\
\text { thyroglobulin type-I } \\
\text { domain. }\end{array}$ & $\begin{array}{l}\text { Prolonging the half-life of } \\
\text { insulin-like growth factor } \\
\text { (IGF) and altering their } \\
\text { interaction with cell surface } \\
\text { receptors }\end{array}$ & -0.116 \\
\hline $\begin{array}{l}\text { Notes. } \\
\text { PLK1, } \\
\text { Tyrosine } \\
\text { adenine } \\
\text { Response }\end{array}$ & $\begin{array}{l}\text { olo-like kinase 1; MET, Me } \\
\text { inase 2; LDH/MDH, lactate an } \\
\text { ucleotide; IGFBP, insulin-like } \\
\text { NADPH, nicotinamide adenin }\end{array}$ & $\begin{array}{l}\text { senchymal Epithelial Trans } \\
\text { Malate dehydrogenases; N } \\
\text { growth factor-binding prot } \\
\text { e dinucleotide phosphate. }\end{array}$ & $\begin{array}{l}\text { ion; MEF } \\
\text { D, Nicoti } \\
\text { n; IGF, }\end{array}$ & $\begin{array}{l}\text { AXL/TYRO3 receptor, } \\
\text { Imide adenine dinucleoti } \\
\text { sulin-like growth factor }\end{array}$ & $\begin{array}{l}\text { TAM receptors; TYK2, } \\
\text { de; NADH, Nicotinamide } \\
\text { IER3, Immediate Early }\end{array}$ & \\
\hline
\end{tabular}




\section{Table 2 (on next page)}

Clinical characteristics and risk score of HCC utilizing multivariate cox regression in TCGA dataset. 


\begin{tabular}{llllllll}
\hline Variable & $\mathrm{B}$ & $\mathrm{SE}$ & $\mathrm{z}$ & $\mathrm{HR}$ & HR.95L & HR.95H & $P$ value \\
\hline age & 0.001 & 0.007 & 0.205 & 1.001 & 0.987 & 1.016 & 0.837 \\
gender & 0.087 & 0.204 & 0.425 & 1.091 & 0.731 & 1.627 & 0.671 \\
grade & -0.141 & 0.150 & -0.942 & 0.868 & 0.647 & 1.165 & 0.346 \\
stage & 0.334 & 0.117 & 2.853 & 1.397 & 1.110 & 1.757 & 0.004 \\
Risk Score & 1.164 & 0.171 & 6.824 & 3.204 & 2.293 & 4.476 & $<0.001$ \\
\hline
\end{tabular}

